\title{
Antidepressants use and risk of cataract development: a systematic review and meta-analysis
}

\author{
Yana Fu', Qi Dai ${ }^{1 *} \mathbb{D}$, Liwei Zhu² and Shuangqing $\mathrm{Wu}^{2}$
}

\begin{abstract}
Background: Epidemiological studies suggest that antidepressants use may increase the risk of cataract, but the results are inconclusive. We aimed to examine this association by performing a systematic review and meta-analysis.

Methods: Relevant studies were identified by searching PubMed and Web of Science databases through June 2017. We included studies that reported risk estimates for the association between antidepressants use and cataract risk. A random-effects model was used to calculate the summary odds ratio (OR) with its $95 \%$ confidence interval (Cl).

Results: We identified seven studies of antidepressants use and risk of cataract involving 447,672 cases and 1,510,391 controls. Overall, the combined ORs ( $95 \% \mathrm{Cls}$ ) of cataract for selective serotonin reuptake inhibitors (SSRIs), serotonin noradrenalin reuptake inhibitors (SNRIs), and tricyclic antidepressants (TCAs) were 1.12 (1.06-1.19), 1.13 (1.04-1.24), and $1.19(1.11-1.28)$, respectively. A certain degree of heterogeneity was observed across studies $\left(P<0.001, P^{2}=92.2 \%\right.$ for SSRIs, $P=0.026, P^{2}=67.5 \%$ for SNRIs, and $P=0.092, P^{2}=58.0 \%$ for TCAs).

Conclusion: This meta-analysis provides evidence of a significant positive association between antidepressants use and risk of cataract. Because of the heterogeneity and limited eligible studies, further prospective studies are warranted to confirm the preliminary findings of our study.
\end{abstract}

Keywords: Antidepressants, Cataract, Meta-analysis, Risk

\section{Background}

Cataract is defined as partial or complete loss of transparency of the crystalline lens and is considered the primary cause of vision loss worldwide [1]. The high prevalence and incidence of cataract have resulted in a large public health burden. Although the actual mechanism of cataract development remains unclear, several risk factors have been established, including age [2], corticosteroid use [3], hypertension [4], smoking [5], and so on.

Recently, emerging epidemiological studies have focused on the risk of cataract formation of antidepressants. Two population-based studies from Canada [6] and the United States [7] suggested a significant positive association between the use of selective serotonin reuptake inhibitors (SSRIs) and the incidence of cataract.

\footnotetext{
* Correspondence: dq@mail.eye.ac.cn

${ }^{1}$ The Eye Hospital of Wenzhou Medical University, 270 Xueyuan West Road, Wenzhou City 325027, Zhejiang Province, People's Republic of China Full list of author information is available at the end of the article
}

Beaver Dam Eye study showed a tendency toward an increased risk of cataract in users of amitriptyline, a tricyclic antidepressant (TCA) [8]. On the other hand, Becker et al. [9] failed to find a positive relationship between SSRIs and cataract risk using the UK-based Clinical Practice Research Datalink (CPRD).

Given the inconsistency and conflict of the existing literature and the insufficient statistical power of individual studies, we performed the present meta-analysis based on all eligible epidemiological studies that provided data on the association of antidepressants use with cataract risk.

\section{Methods \\ Literature search \\ We performed this meta-analysis in accordance with the Meta-Analysis of Observational Studies in Epidemiology guidelines [10]. A systematic literature search was car- ried out in PubMed and Web of Science databases through June 2017 by using the following search}


strategy: ("antidepressant" or "depression" or "selective serotonin reuptake inhibitor" or "SSRI" or "monoaminoxidase inhibitor" or "MAOI" or "tricyclic antidepressant" or "TCA" or "serotonin noradrenalin reuptake inhibitor" or "SNRI" or "serotonin antagonist and reuptake inhibitor" or "SARI" or "norepinephrine dopamine reuptake inhibitor" or "NDRI" or "norepinephrine reuptake inhibitor" or "NRI" or "noradrenergic and specific serotonergic antidepressant" or "NaSSA") and "Cataract", with no restrictions. Cited references of the retrieved articles and reviews were also checked.

\section{Study selection}

Studies included in this meta-analysis met the following criteria: 1) had cohort, nested case-control or casecontrol study design; 2) the exposure of interest was antidepressants use, including SSRIs, TCAs, serotonin noradrenalin reuptake inhibitors (SNRIs), and so on; 3) the endpoint of interest was cataract incidence; and 4) the risk estimate and the corresponding 95\% confidence interval $(\mathrm{CI})$ were reported. If multiple studies used the same population, we included the study with the largest sample size.

\section{Data extraction and quality assessment}

We extracted the following data using a standardized data-collection form: last name of the first author, publication year, study region, number of cases and controls, method of exposure and endpoint assessment, types of antidepressants, risk estimates from the most fully adjusted model and the corresponding 95\% CIs, matched or adjusted potential confounders.

We assessed the quality of individual studies using Newcastle-Ottawa Scale (NOS), a 9-star system which consists of three dimensions: selection (four items), comparability (one item), and exposure/outcome (three items) (http://www.ohri.ca/programs/clinical_epidemiology/ oxford.asp). Two authors (QD and YF) independently performed the literature search, study selection, data extraction, and quality assessment. Any disagreements were resolved by consensus.

\section{Statistical analysis}

A DerSimonian and Laird random-effects model [11], which considered both within- and between-study variation, was used to calculate the combined estimate of effect size. For studies that separately provided estimated risk estimates for a number of categories of exposure compared with a single reference category, we combined these risk estimates within each study using the method reported by Hamling et al.'s study [12]. Homogeneity across included studies was tested by $Q$ statistics at the $P<0.10$ level of significance [13]. The $I^{2}$ score, a quantitative measure of inconsistency across studies, was also calculated [13]. Potential publication bias was assessed by a visual funnel plot [14]. All analyses were performed by using STATA version 10.0 (StataCorp, College Station, TX). A $P$ value $<0.05$ was considered statistically significant, except where otherwise specified.

\section{Results}

Literature search and study characteristics

A flow chart showing the study selection process in detail is presented in Fig. 1. Seven studies [6-9, 15-17] were finally included in this meta-analysis. Of these, five studies were performed in North America, one in Europe, and one in Asia. All individual studies were case-control studies, of which four were nested case-

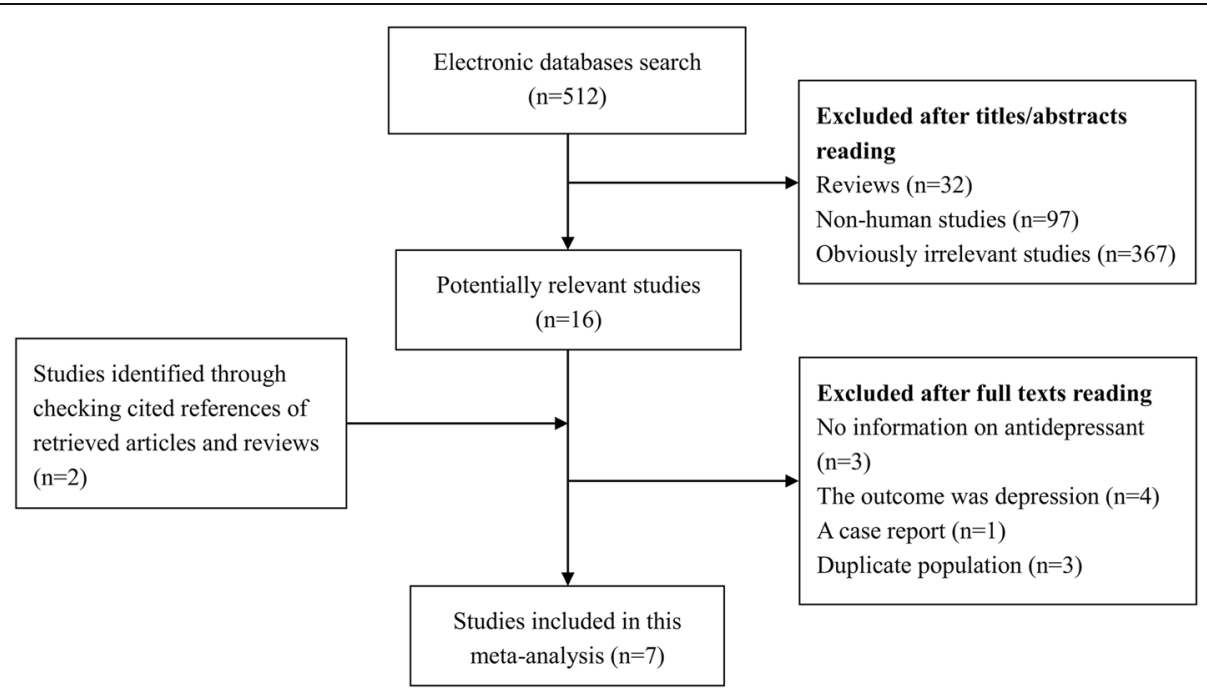

Fig. 1 Flow chart of study selection 


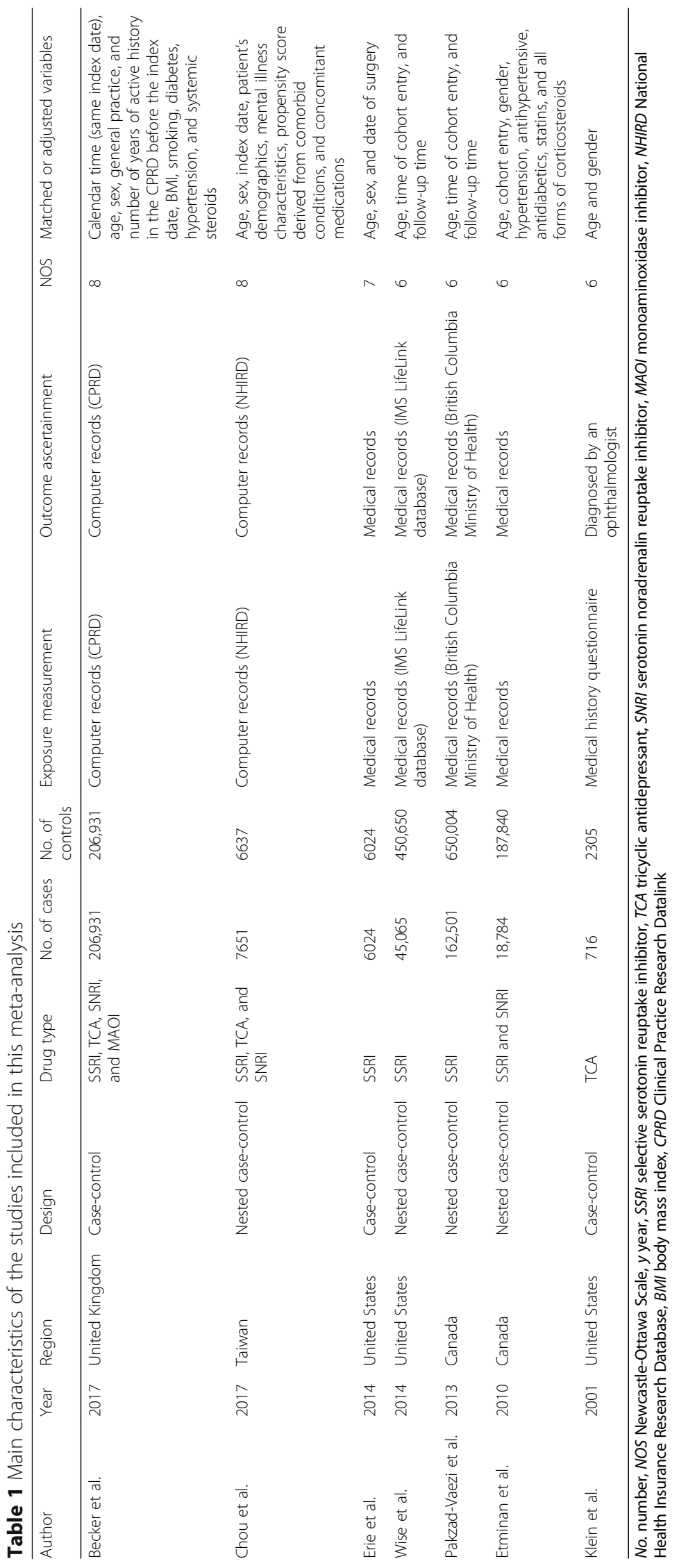



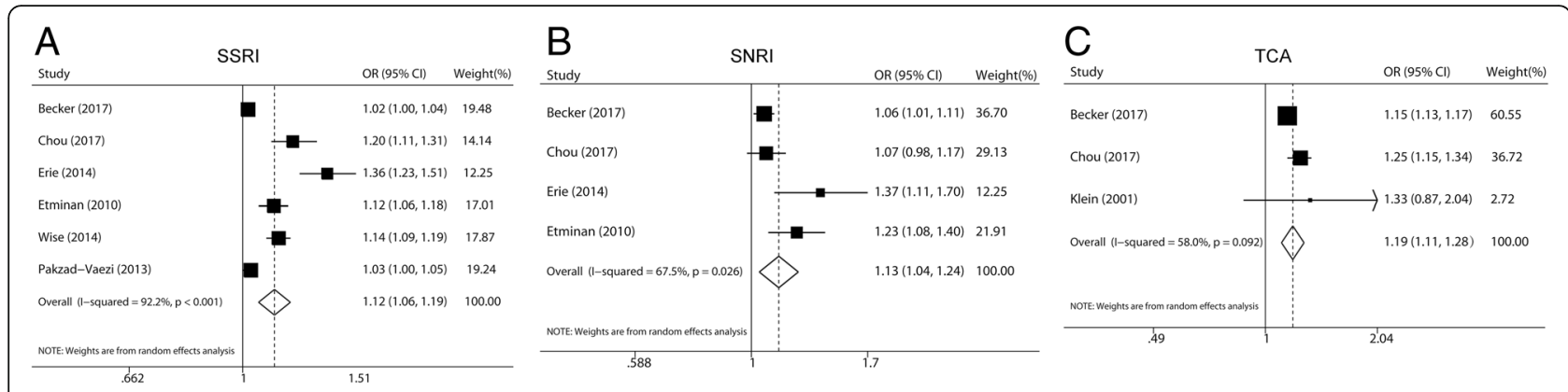

Fig. 2 Random-effects meta-analysis of studies that examined three types of antidepressants use may increase the risk of cataract. a SSRIs use; b SNRIs use; c TCAs use

control studies. Results for SSRIs were presented in six of these studies, four for SNRIs, and three for TCAs. These studies were published between 2001 and 2017. Information on exposure and endpoint were mainly collected from medical records. The NOS scores ranged from six to eight, with a mean value of 6.7. The main characteristics of all included studies have been summarized in Table 1 .

\section{Main analysis by antidepressant classifications}

The multivariable-adjusted odds ratios (ORs) for each study and the pooled ORs for the any exposure versus none of antidepressants are presented in Fig. 2. Overall, the combined ORs (95\% CIs) of cataract for SSRIs, SNRIs, and TCAs were 1.12 (1.06-1.19), 1.13 (1.04-1.24), and 1.19 (1.11-1.28), respectively. Obvious heterogeneity was found across studies $\left(P<0.001, I^{2}=92.2 \%\right.$ for SSRIs, $P=0.026, I^{2}=67.5 \%$ for SNRIs, and $P=0.092$, $I^{2}=58.0 \%$ for TCAs).
Subgroup analyses by individual antidepressant drugs

The results of subgroup analysis according to individual antidepressant drugs are presented in Fig. 3. For SSRIs antidepressant drugs, a significantly direct association with cataract incidence was observed for fluoxetine (RR 1.08, 95\% CI 1.03-1.12) and fluvoxamine (RR 1.22, 95\% CI 1.06-1.40). No evidence of association was found for the rest of SSRIs drugs. For SNRIs antidepressant drugs, the combined ORs (95\% CIs) of cataract for any exposure versus none were 1.35 (0.77-2.36), 2.33 (0.94-5.74), and 1.30 (1.18-1.43) for duloxetine, milnacipran, and venlafaxine, respectively.

\section{Publication bias}

As only seven studies were included in the present meta-analysis, Begg's test [18] and Egger's test [14] were not eligible for publication bias analysis. Hence we adopted a visual funnel plot to qualitatively assess the publication bias. As shown in Fig. 4, a certain degree of

\begin{tabular}{|c|c|c|c|c|c|}
\hline Drug & Q & $\mathrm{P}$ & $1^{2}$ & & OR $(95 \% \mathrm{Cl})$ \\
\hline \multicolumn{6}{|l|}{ SSRI } \\
\hline Citalopram & 11.32 & 0.003 & $82.30 \%$ & E. & $1.25(1.00,1.56)$ \\
\hline Fluoxetine & 3.1 & 0.377 & $3.20 \%$ & + & $1.08(1.03,1.12)$ \\
\hline Paroxetine & 6.9 & 0.075 & $56.50 \%$ & t & $1.05(0.96,1.15)$ \\
\hline Sertraline & 10.99 & 0.012 & $72.70 \%$ & + & $1.11(0.99,1.25)$ \\
\hline Fluvoxamine & 2.39 & 0.495 & $0.00 \%$ & $\rightarrow$ & $1.22(1.06,1.40)$ \\
\hline Escitalopram & 0.02 & 0.884 & $0.00 \%$ & 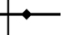 & $1.14(0.90,1.45)$ \\
\hline \multicolumn{6}{|l|}{ SNRI } \\
\hline Duloxetine & 2.56 & 0.11 & $60.90 \%$ & $\rightarrow$ & $1.35(0.77,2.36)$ \\
\hline Milnacipran & NA & NA & NA & & $2.33(0.94,5.74)$ \\
\hline Venlafaxine & 1.83 & 0.401 & $0.00 \%$ & $\rightarrow$ & $1.30(1.18,1.43)$ \\
\hline & & 17 & & 1 & \\
\hline
\end{tabular}

Fig. 3 Subgroup analyses by individual antidepressant drugs 


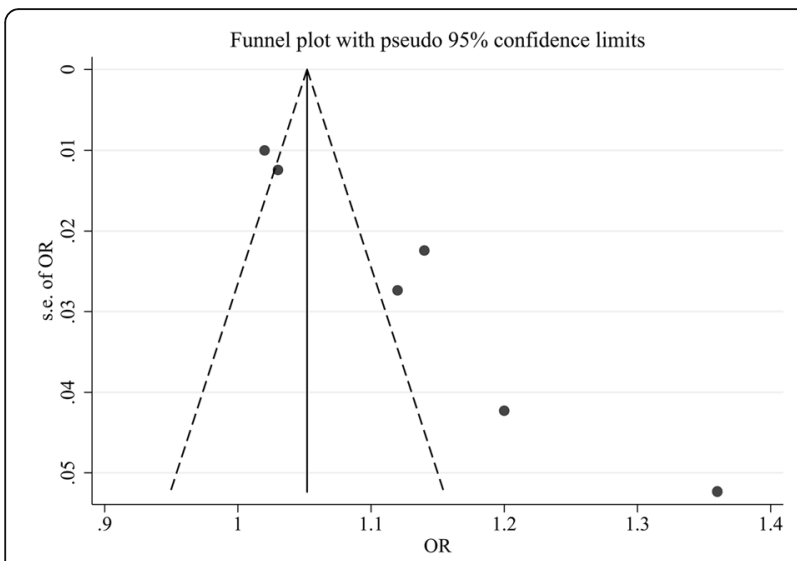

Fig. 4 A visual funnel plot to qualitatively assess the publication bias

asymmetry was observed, which indicated slight publication bias.

\section{Discussion}

This meta-analysis of seven eligible studies involving 447,672 cases and 1,510,391 controls supports a significant positive association of SSRIs, SNRIs and TCAs use with risk of cataract. To the best of our knowledge, this is the first systematic review and meta-analysis aimed to evaluate the relationship between antidepressants use and risk of cataract development.

Heterogeneity is often a concern in a meta-analysis. In the present study, obvious heterogeneity was observed among most analyses, which was partially explained by the following factors: study design was different. Although most included studies were performed in Western countries, population characteristics still varied in genetic and environmental background, antidepressants use, and matched or adjusted confounders.

Several mechanisms may be involved in the positive association of antidepressants use with cataract risk. In animal models, serotonin has been reported to play a crucial role in lens transparency [19]. Elevated serotonin levels have been shown to lead to lens opacity in rats [20]. Similarly, cataract and glaucoma patients also had increased levels of serotonin in the aqueous humor [21]. In addition, serotonin 5-HT1A, 5-HT2A/2C, and 5-HT7 receptors have been identified in the crystalline lens, which participate in regulation of intraocular pressure (IOP) homeostasis [22]. Increased IOP is able to contribute to glaucoma, which is a risk factor for cataract formation [23]. TCAs use is reported to be related with photosensitivity to ultraviolet or sunlight. This latter exposure has been suggested to be associated with cortical cataract in Beaver Dam Eye Study [24]. On the other hand, TCAs is able to inhibit norepinephrine uptake, which may also have cataractogenic properties [25].
Our study had some important strengths. Considering individual studies had limited statistical power, this meta-analysis of seven studies involving a large number of cases and controls improved the power to detect a potential association and provided more robust estimates. Most of the original studies matched or adjusted a series of variables, which greatly reduced the likelihood of confounding bias.

Potential limitations of our study should be considered. First, the number of eligible studies was limited, especially in some subgroup analyses, which might influence the reliability of the results. Second, significant heterogeneity was observed among included studies, which might distort the conclusion of our study. Third, a certain degree of publication bias was observed. Gray literature (e.g., conference abstract) was difficult to find and studies with null results were less likely to be published. Finally, random misclassification of antidepressants might influence the results.

\section{Conclusion}

Use of antidepressants, including SSRIs, SNRIs and TCAs, is associated with an increased risk of cataract development. Considering the huge heterogeneity and limited included studies, further large well-designed prospective studies are warranted to confirm the preliminary findings of our study.

\section{Abbreviations \\ 95\% Cl: 95\% Confidence Interval; NOS: Newcastle-Ottawa Scale; OR: Odds Ratio; SNRIs: Serotonin noradrenalin reuptake inhibitors; SSRIs: Selective serotonin reuptake inhibitors; TCAs: Tricyclic antidepressants}

\section{Acknowledgements}

Not Applicable.

\section{Funding}

This study was supported by grants from the Natural Science Foundation of Zhejiang Province (LY17H120009, LY17H120008).

\section{Availability of data and materials}

The data used in our study can be found in corresponding published literatures. Please see the references in the manuscript.

\section{Authors' contributions}

$Q D, Y F, S W$ and $L Z$ gave work in the process of designing the study, revising and deciding the final edition of the manuscript. YF and $L Z$ were in charge of data collection, analysis and drafting the manuscript. SW and LZ provided assistance in the data collection. QD and YF provided aids for literature screening. The content of the final version was read and approved by all the authors.

Ethics approval and consent to participate Not applicable.

Consent for publication Not applicable.

Competing interests

The authors declare that they have no competing interests. 


\section{Publisher's Note}

Springer Nature remains neutral with regard to jurisdictional claims in published maps and institutional affiliations.

\section{Author details}

${ }^{1}$ The Eye Hospital of Wenzhou Medical University, 270 Xueyuan West Road, Wenzhou City 325027, Zhejiang Province, People's Republic of China. 2Department of Ophthalmology, Hangzhou Red Cross Hospital, Hangzhou City 310003, Zhejiang Province, People's Republic of China.

Received: 10 November 2017 Accepted: 30 January 2018 Published online: 06 February 2018

\section{References}

1. Bourne RR, Stevens GA, White RA, Smith JL, Flaxman SR, Price $H$, et al. Causes of vision loss worldwide, 1990-2010: a systematic analysis. Lancet Glob Health. 2013;1(6):e339-49.

2. Mitchell P, Cumming RG, Attebo K, Panchapakesan J. Prevalence of cataract in Australia: the Blue Mountains eye study. Ophthalmology. 1997;104(4):581-8.

3. Weatherall M, Clay J, James K, Perrin K, Shirtcliffe P, Beasley R. Dose-response relationship of inhaled corticosteroids and cataracts: a systematic review and meta-analysis. Respirology. 2009;14(7):983-90.

4. Yu X, Lyu D, Dong X, He J, Yao K. Hypertension and risk of cataract: a meta-analysis. PLoS One. 2014;9(12):e114012.

5. Ye J, He J, Wang C, Wu H, Shi X, Zhang H, et al. Smoking and risk of age-related cataract: a meta-analysis. Invest Ophthalmol Vis Sci. 2012;53(7):3885-95.

6. Etminan M, Mikelberg FS, Brophy JM. Selective serotonin reuptake inhibitors and the risk of cataracts: a nested case-control study. Ophthalmology. 2010;117(6):1251-5.

7. Erie JC, Brue SM, Chamberlain AM, Hodge DO. Selective serotonin reuptake inhibitor use and increased risk of cataract surgery: a population-based, case-control study. Am J Ophthalmol. 2014;158(1):192-7. el

8. Klein BE, Klein R, Lee KE, Danforth LG. Drug use and five-year incidence of age-related cataracts: the beaver dam eye study. Ophthalmology. 2001:108(9):1670-4.

9. Becker C, Jick SS, Meier CR. Selective Serotonin Reuptake Inhibitors and Cataract Risk: A Case-Control Analysis. Ophthalmology. 2017;124(11):1635-9.

10. Moher D, Liberati A, Tetzlaff J, Altman DG. Preferred reporting items for systematic reviews and meta-analyses: the PRISMA statement. Ann Intern Med. 2009;151(4):264-9. W64

11. DerSimonian R, Laird N. Meta-analysis in clinical trials. Control Clin Trials. 1986;7(3):177-88.

12. Hamling J, Lee $P$, Weitkunat $R$, Ambuhl M. Facilitating meta-analyses by deriving relative effect and precision estimates for alternative comparisons from a set of estimates presented by exposure level or disease category. Stat Med. 2008:27(7):954-70.

13. Higgins JP, Thompson SG. Quantifying heterogeneity in a meta-analysis. Stat Med. 2002;21(11):1539-58.

14. Egger M, Davey Smith G, Schneider M, Minder C. Bias in meta-analysis detected by a simple, graphical test. BMJ. 1997:315(7109):629-34.

15. Pakzad-Vaezi KL, Etminan M, Mikelberg FS. The association between cataract surgery and atypical antipsychotic use: a nested case-control study. Am J Ophthalmol. 2013;156(6):1141-6. e1

16. Wise SJ, Nathoo NA, Etminan M, Mikelberg FS, Mancini GB. Statin use and risk for cataract: a nested case-control study of 2 populations in Canada and the United States. Can J Cardiol. 2014;30(12):1613-9.

17. Chou PH, Chu CS, Chen YH, Hsu MY, Huang MW, Lan TH, et al. Antidepressants and risk of cataract development: a population-based, nested case-control study. J Affect Disord. 2017:215:237-44.

18. Begg CB, Mazumdar M. Operating characteristics of a rank correlation test for publication bias. Biometrics. 1994;50(4):1088-101.

19. Vivekanandan S, Lou MF. Evidence for the presence of phosphoinositide cycle and its involvement in cellular signal transduction in the rabbit lens. Curr Eye Res. 1989;8(1):101-11.

20. Boerrigter RM, Siertsema JV, Kema IP. Serotonin (5-HT) and the rat's eye. Some pilot studies. Doc Ophthalmol. 1992:82(1-2):141-50.

21. Zanon-Moreno V, Melo P, Mendes-Pinto MM, Alves CJ, Garcia-Medina J J Vinuesa-Silva I, et al. Serotonin levels in aqueous humor of patients with primary open-angle glaucoma. Mol Vis. 2008;14:2143-7.

22. Costagliola C, Parmeggiani F, Sebastiani A. SSRIs and intraocular pressure modifications. CNS Drugs. 2004;18(8):475-84.
23. Hodge WG, Whitcher JP, Satariano W. Risk factors for age-related cataracts Epidemiol Rev. 1995;17(2):336-46.

24. Cruickshanks KJ, Klein BE, Klein R. Ultraviolet light exposure and lens opacities: the beaver dam eye study. Am J Public Health. 1992;82(12):1658-62.

25. Trope GE, Sole M, Aedy L, Madapallimattam A. Levels of norepinephrine, epinephrine, dopamine, serotonin and $\mathrm{N}$-acetylserotonin in aqueous humour. Can J Ophthalmol. 1987;22(3):152-4.

\section{Submit your next manuscript to BioMed Central and we will help you at every step:}

- We accept pre-submission inquiries

- Our selector tool helps you to find the most relevant journal

- We provide round the clock customer support

- Convenient online submission

- Thorough peer review

- Inclusion in PubMed and all major indexing services

- Maximum visibility for your research

Submit your manuscript at www.biomedcentral.com/submit
Biomed Central 